PACS 85.60.-q, Dw, Jb

\title{
Dynamic characteristics of QWIP-HBT-LED optoelectronic integrated devices
}

\author{
Sh.M. Eladl, A. Nasr, and A. Aboshosha \\ Radiation Engineering Department, 3 Ahmed Elzomor Str., NCRRT, P.O. Box 29, \\ Nasr City, Atomic Energy Authority, Cairo, Egypt
}

\begin{abstract}
This paper presents an evaluation of the transient performance of an optoelectronic integrated device. This device is composed of a quantum well infrared photodetector (QWIP), a heterojunction bipolar transistor (HBT) and a light emitting diode (LED). It is called as QWIP-HBT-LED optoelectronic integrated device. Evaluation of its transient response is based on the frequency response of the constituent devices. Analytical expressions describing the transient behavior, output derivative as a measure of speed, and the rise time are derived. The numerical results show that the transient performance of the version under consideration is mainly based on the individual quantum efficiencies and is improved with their growth. The device speed and rise time are enhanced with the increase of the cut-off frequency of HBT.
\end{abstract}

Keywords: optoelectronic integrated device, quantum well infrared photodetector, light emitting diode, heterojunction bipolar transistor.

Manuscript received 30.03.09; accepted for publication 14.05.09; published online 15.05.09.

\section{Introduction}

For optical image processing applications, it is necessary to focus on devices and components that can detect, process, and transmit information with great adaptability and better efficiency [1]. Integration of a quantum well infrared photodetector (QWIP) and a light emitting diode (LED) is an effective method to obtain optoelectronic integrated device that can be used as a pixel sensitive to far or middle infrared radiation with near infrared output [2]. The previous studies focused on fabrication and analysis of the static device performance, however, they did not analyze the transient behavior of the device [3-5].

Recently [6], evaluation of a novel device based on integration of QWIP, heterojunction bipolar transistor (HBT), and LED for up-conversion of middle infrared into near infrared (visible) radiation has been presented. It was shown that the external quantum efficiency can be of the order of unity that can provide significant advantages of QWIP-HBT-LED based focal plane arrays (FPAs) over the FPAs of other types. The effect of interface recombination and self-absorption within the LED active region on the efficiency of QWIP-HBT-LED integrated device was studied by [7]. It was observed that the quantum conversion efficiency of the device under consideration was lowered for self-absorption and interface recombination within the recombination region of the LED.

Operation of this device can be explained as follows: the input light is converted to photogenerated carriers through the QWIP, the QWIP output electric signal is amplified by the HBT and the LED is driven by the output amplified signal injected from the HBT and emits an intensified light of near infrared or visible radiation.

Fig. 1 shows the schematic layer structure of the device under study. The paper is organized as follows. Formulation of the specified parameters that describe the transient response, derivative, and rise time is presented in this section. The generated curves as results are outlined and discussed in Section 3. Finally, conclusion of the work and some important notes for the continuity of the research in this subject have been discussed in Section 4 .

\section{Theoretical analysis}

To understand the transient response of the device version under study, it is important to investigate the transient response of each element that constitutes it. The recognition of the characteristic equation describing the overall frequency response becomes available when the frequency response equation of each element is known. 


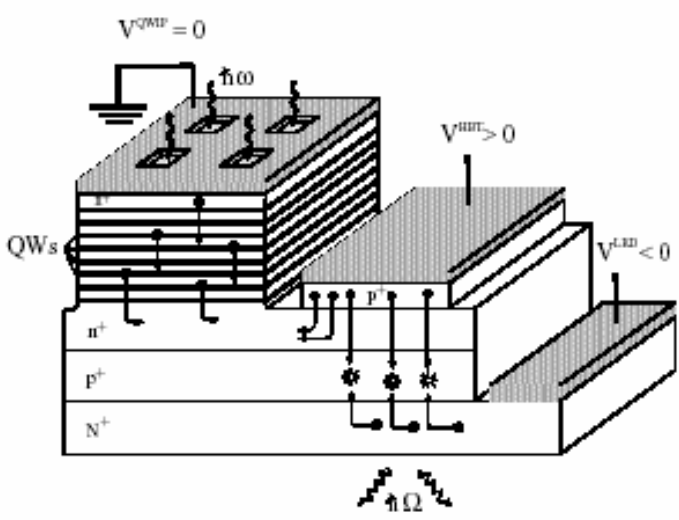

Fig. 1. Schematic structure of QWIP-HBT-LED device [6].

With account of the frequency response for the constituent devices described as in Refs $[8,9]$, the frequency response of QWIP-HBT-LED can be derived as:

$$
\begin{array}{r}
\eta_{c}(\omega)=\eta_{\mathrm{QWIP}}(\omega) \eta_{\mathrm{HBT}}(\omega) \eta_{\mathrm{LED}}(\omega)= \\
\frac{\eta_{\mathrm{QWIP}} \eta_{\mathrm{HBT}} \eta_{\mathrm{LED}}}{\left(1+\frac{J \omega}{\omega_{\mathrm{QWIP}}}\right)\left(1+\frac{J \omega}{\omega_{\mathrm{HBT}}}\right)\left(1+\frac{J \omega}{\omega_{\mathrm{LED}}}\right)} .
\end{array}
$$

Here, $\eta_{\mathrm{QWIP}}(\omega), \eta_{\mathrm{HBT}}(\omega)$, and $\eta_{\mathrm{LED}}(\omega)$ are the quantum efficiencies for QWIP, HBT, and LED, respectively. Also, $\omega_{\mathrm{QWIP}}, \omega_{\mathrm{HBT}}$, and $\omega_{\mathrm{LED}}$ are the cutoff frequency of QWIP, HBT, and LED, respectively, and $\omega$ is frequency of the incident infrared image.

The Laplace transform of the above equation can be expressed as:

$$
\begin{aligned}
& \eta(t)=\eta_{\mathrm{QWIP}} \eta_{\mathrm{HBT}} \eta_{\mathrm{LED}} \times \\
& \times\left(\begin{array}{c}
1-\frac{\omega_{\mathrm{LED}} \omega_{\mathrm{HBT}} e^{-\omega_{\mathrm{QWIP}} t}}{\left(\omega_{\mathrm{LED}}-\omega_{\mathrm{QWIP}}\right)\left(\omega_{\mathrm{HBT}}-\omega_{\mathrm{QWIP}}\right)} \\
-\frac{\omega_{\mathrm{LED}} \omega_{\mathrm{QWIP}} e^{-\omega_{\mathrm{HBT}} t}}{\left(\omega_{\mathrm{HBT}}-\omega_{\mathrm{LED}}\right)\left(\omega_{\mathrm{HBT}}-\omega_{\mathrm{QWIP}}\right)} \\
+\frac{\omega_{\mathrm{QWIP}} \omega_{\mathrm{HBT}} e^{-\omega_{\mathrm{LED}} t}}{\left(\omega_{\mathrm{LED}}-\omega_{\mathrm{QWIP}}\right)\left(\omega_{\mathrm{HBT}}-\omega_{\mathrm{LED}}\right)}
\end{array}\right) .
\end{aligned}
$$

The final state quantum efficiency of the structure can be expressed as in Ref. [6]

$$
\eta=\eta_{\mathrm{QWIP}} \eta_{\mathrm{HBT}} \eta_{\mathrm{LED}}
$$

The derivative of the quantum efficiency of the device with respect to time denoted by $v$ is expressed by $\mathrm{v}(t)=\frac{d \eta(t)}{d t}$, which describes how fast the quantum efficiency changes with time, this quantity can be expressed as:

$$
\frac{d \eta(t)}{d t}=\zeta \times\left(\begin{array}{c}
\frac{e^{-\omega_{\mathrm{QWIP}} t}}{\left(\omega_{\mathrm{QWIP}}-\omega_{\mathrm{LED}}\right)\left(\omega_{\mathrm{QWIP}}-\omega_{\mathrm{HBT}}\right)} \\
-\frac{e^{-\omega_{\mathrm{HBT}} t}}{\left(\omega_{\mathrm{HBT}}-\omega_{\mathrm{LED}}\right)\left(\omega_{\mathrm{QWIP}}-\omega_{\mathrm{HBT}}\right)} \\
+\frac{e^{-\omega_{\mathrm{LED}} t}}{\left(\omega_{\mathrm{QWIP}}-\omega_{\mathrm{LED}}\right)\left(\omega_{\mathrm{HBT}}-\omega_{\mathrm{LED}}\right)}
\end{array}\right),
$$

where

$\zeta=\eta_{\mathrm{QWIP}} \eta_{\mathrm{HBT}} \eta_{\mathrm{LED}} \omega_{\mathrm{QWIP}} \omega_{\mathrm{HBT}} \omega_{\mathrm{LED}} \cdot$

The rise time of the QWIP-HBT-LED is the time needed for the quantum efficiency to reach the final value $\eta_{f}$ of the quantum efficiency in the final state. By using the approximation $\omega_{\mathrm{HBT}}<\omega_{\mathrm{QWIP}}<\omega_{\mathrm{LED}}$, the rise time

$$
R_{t}=\frac{-1}{\omega_{\mathrm{HBT}}} \ln \left[\frac{D}{\eta_{\mathrm{QWIP}} \eta_{\mathrm{HBT}} \eta_{\mathrm{LED}} \omega_{\mathrm{QWIP}} \omega_{\mathrm{HBT}} \omega_{\mathrm{LED}}}\right],
$$

where

$D=\left\lfloor-\eta_{\mathrm{QWIP}} \eta_{\mathrm{HBT}} \eta_{\mathrm{LED}} \omega_{\mathrm{QWIP}} \omega_{\mathrm{HBT}}\right\rfloor+$

$+\eta_{\mathrm{QWIP}} \eta_{\mathrm{HBT}} \eta_{\mathrm{LED}} \omega_{\mathrm{QWIP}} \omega_{\mathrm{LED}}+$

$+\eta_{\mathrm{QWIP}} \eta_{\mathrm{HBT}} \eta_{\mathrm{LED}} \omega_{\mathrm{HBT}}^{2}-\eta_{\mathrm{QWIP}} \eta_{\mathrm{HBT}} \eta_{\mathrm{LED}} \omega_{\mathrm{HBT}} \omega_{\mathrm{LED}}+$ $+\eta_{f}\left[\omega_{\mathrm{QWIP}} \omega_{\mathrm{HBT}}-\omega_{\mathrm{QWIP}} \omega_{\mathrm{LED}}+\omega_{\mathrm{LED}} \omega_{\mathrm{HBT}}-\omega_{\mathrm{HBT}}^{2}\right]$.

\section{Results and discussions}

The device parameters used in the following calculations are as follows: $\omega_{\mathrm{HBT}}=10^{8} \mathrm{~Hz}, \quad \omega_{\mathrm{QWIP}}=10^{9} \mathrm{~Hz}$, $\omega_{\text {LED }}=10^{10} \mathrm{~Hz}$, and $\eta_{\mathrm{QWIP}} \eta_{\mathrm{HBT}} \eta_{\mathrm{LED}}=0.6$. The input infrared radiation is assumed to be a step function in time. The transient response of the quantum efficiency of QWIP-HBT-LED device is shown in Fig. 2. It can be seen that the quantum efficiency increases with time until approaches a definite and stable value. This value is dependent on the values of the quantum efficiencies of the constituent devices. The cut-off frequency of HBT has the dominant effect on the arrival time to this definite value, while the cut-off frequency for both QWIP and LED has no effect on this arrival time.

Fig. 3 shows the time dependence of the derivative of the quantum conversion (which describes how fast the quantum efficiency changes with time) at different values of $\eta_{\mathrm{HBT}}$, it can be seen that the output derivative increases with the increase of $\eta_{\text {HBT }}$ at any time. At early time, the quantum efficiency changes with an increasing derivative until arrives a peak value and begins to decrease in derivative with time after that. This is due to the cut-off frequency of HBT is lower than those of both 


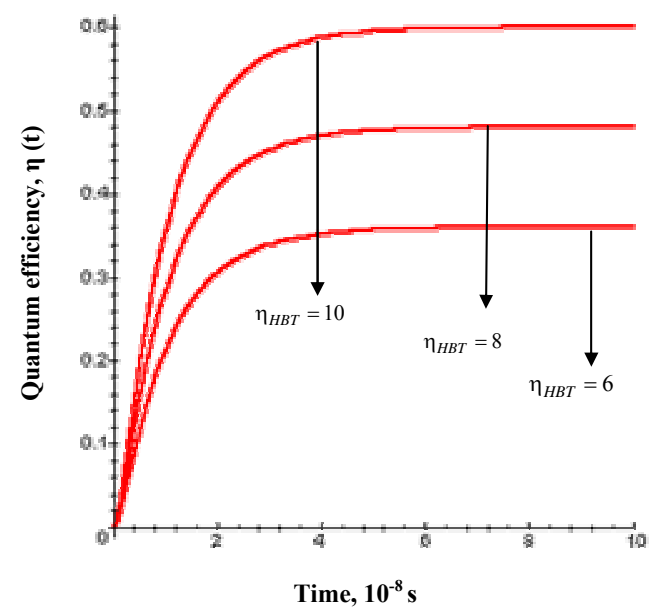

Fig. 2. Dynamic response of QWIP-HBT-LED integrated device.

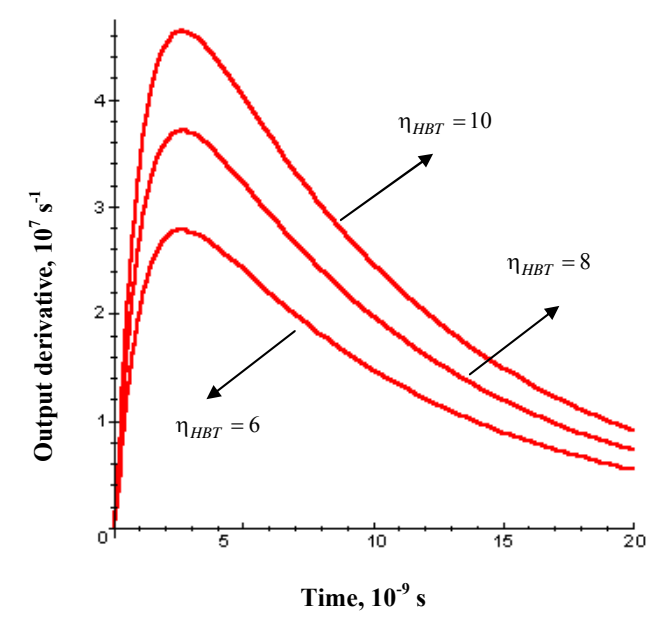

Fig. 3. Variation of quantum efficiency derivative with time.

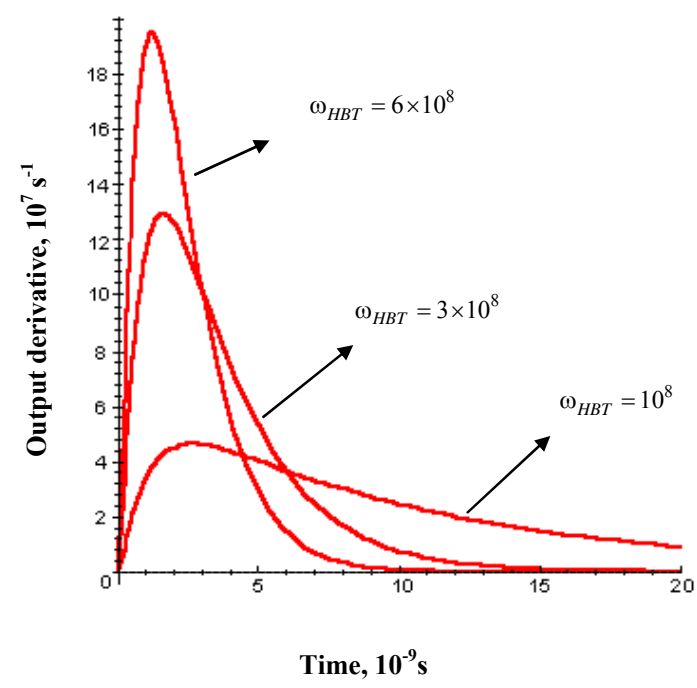

Fig. 4. Dependence of the output derivative on time.

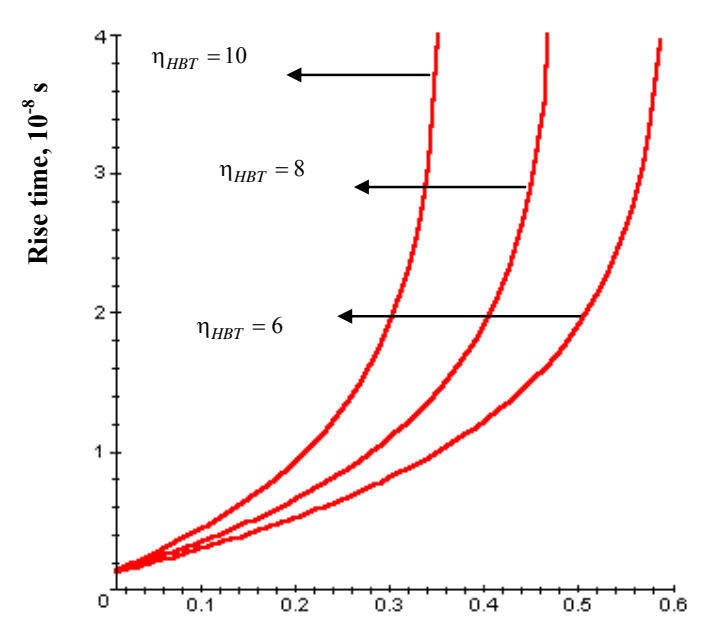

Fig. 5. Rise time versus quantum efficiency at different $\eta_{\text {HBT }}$ values.

QWIP and LED. While Fig. 4 shows the time dependence of the derivative of the quantum conversion at different values of $\omega_{\mathrm{HBT}}$, it can be seen that the cutoff frequency of HBT has a major effect on the behavior of the quantum conversion derivative, as it is rapidly decreased at higher values of $\omega_{\mathrm{HBT}}$, while $\omega_{\mathrm{HBT}}$ has no effect on the arrival time peak value of output derivative.

The dependence of the rise time of the device on the quantum efficiency is shown in Fig. 5. It is clear from this figure that, as the final value of quantum efficiency is increased, the rise time to this value is also increased, because the difference between the initial and final values is increased, which requires more time to reach this final value and hence the increase in rise time of the device. Also, the quantum efficiency of HBT causes an enhancement of the output, and rise time is increased as a result.

\section{Conclusion and future work}

Analytical modeling of the transient performance of an optoelectronic integrated device is presented. The device under study is composed of a quantum well infrared photodetector, a heterojunction bipolar transistor and a light emitting diode. The modeling is based on the frequency response of the constituent devices. The analytical expressions describing the transient performance are derived. The results show that the quantum efficiency of HBT causes an enhancement of the output, and rise time is increased as a result. The cutoff frequency of HBT has a major effect on the behavior of the quantum conversion derivative, as it rapidly decreased at higher values of $\omega_{\mathrm{HBT}}$, while it has no effect on the arrival time peak value of output derivative. Also, the cut-off frequency of QWIP and LED has no effect on both the rise time and the output derivative of the device. As a future extension to this study, modeling of this device by the use of its equivalent circuit is 
planned to show the effect of all interesting parameters on the static characteristics.

\section{References}

1. O. Qasaimeh, W. Zhou, P. Bhattacharya et al., Monolithically integrated low-power phototransceivers for optoelectronic parallel sensing and processing applications // J. Lightwave Technology 19, No. 4, p. 546-552 (2001).

2. V. Ryzhii, H.C. Liu, I. Khmyrova, and M. Ryzhii, Analysis of integrated quantum-well infrared photodetector and light-emitting diode for implementing pixelless imaging device // IEEE J. Quantum Electron. 33, No. 9, p. 1527-1531 (1997).

3. L.B. Allard, H.C. Liu, M. Buchanan, and Z.R. Wasilewski, Pixelless infrared imaging utilizing a $\mathrm{p}$ type quantum well infrared photodetector integrated with a light emitting diode // Appl. Phys. Lett. 70, No. 21, p. 2784-2786 (1997).

4. V. Ryzhii, I. Khmyrova, and Ph. Bois, Photon mechanism of image smearing in integrated QWIP LED pixelless devices // IEEE J. Quantum Electron. 35, No. 11, p. 1693-1696 (1999).
5. E. Dupont, M. Byloos, M. Gao, M. Buchanan, C.-Y. Song, Z.R. Wasilewski, and H.C. Liu, Pixelless thermal imaging with integrated quantumwell infrared photodetector and light-emitting diode // IEEE Photon. Technol. Lett. 14, No. 2, p. 182-184 (2002).

6. V. Ryzhii, I. Khmyrova, and S. Oktyabrsky, Device model of integrated QWIP-HBT-LED pixel for infrared focal plane arrays // Proc. 32nd European Solid-State Device Research Conference, ESSDERC 2002, Firenze, Italy, September 24-26, 2002, p. 643-646.

7. Sh.M. Eladl, Analysis of interface recombination and self-absorption effect on the performance of QWIP-HBT-LED integrated device // Microelectron. J. 39, No. 12 p. 1649-1653 (2008).

8. B.C. Roy and N.B. Chakrabarti, Gain and frequency response of heterojunction bipolar phototransistor // IEEE Trans. Electron. Devices 34, No. 7, p. 1482-1490 (1987).

9. $\mathrm{Yu} \mathrm{Zhu}$, Susumu Noda, and Akio Sasaki, Theoretical analysis of transient behavior of optoelectronic integrated devices // IEEE Trans. Electron. Devices 42, No. 4, p. 646-648 (1995). 